emergency and the total delay time, symptom onset until CT completion, is only 4 hours (range 2.3-8.3 hours) (Morris DL et al. 2000). Reasons for excessive diagnostic delay in children include their inability to describe symptoms such as headache, sensory or cerebellar symptoms, and difficulty in recognition of some neurologic signs, eg aphasia. Increased awareness of predisposing causes (eg cardiac, sickle cell disease) should lead to earlier diagnosis and treatment.

Risks of posterior circulation stroke are evaluated in 22 cases (17 boys) identified in a retrospective study at University College and Great Ormond Street Hospitals, London, UK. (Ganesan V, Chong WK, Cox TC et al. Neurology Nov (2 of 2) 2002;59:1552-1556). Vertebrobasilar arterial abnormalities (vertebral artery dissection in 10) were present in 20, multifocal in 12. Cardiac abnormalities with embolism were present in only 4, hypertension in 9, and factor $\mathrm{V}$ Leiden and other gene mutations in 6 . Two had subluxation of the upper cervical spine. During follow-up for 6 months to 11 years, 5 (20\%) had recurrence and 7 had TIA; 12 (50\%) had no residual deficits. Investigations include MRI, cerebral angiography, echocardiography, and cervical radiography. In contrast to anterior circulation stroke, where $50 \%$ have a preexisting disorder, the majority with posterior circulation stroke are previously healthy.

OUTCOME OF HEMISPHERECTOMY IN STURGE-WEBER SYNDROME

A questionnaire was mailed to the parents of 70 patients identified by the Sturge-Weber Foundation as recipients of hemispherectomy between 1979 and 2001, and responses obtained from 32 (46\%) were analysed at the Johns Hopkins Hospital, Baltimore, MD. The mean age at onset of seizures was 4 months, and the median age at surgery was 1.2 years. Eighty one percent were seizure free, and anticonvulsants were discontinued in $53 \%$. Seizure control was not related to the age at seizure onset, but it did correlate with an older age at operation. Six with continued seizures were operated on at 1.3 years, and in 26 currently seizure-free operation was delayed until age 3.1 years $(\mathrm{p}=0.05)$. Hemiparesis was not worsened following surgery. Cognitively, 2 were normal, 10 had mild learning impairment, 14 were moderately learning disabled, and 6 were severely disabled. Cognitive outcome was not related to age at operation or seizure control. (Kossoff EH, Buck C, Freeman JM. Outcomes of 32 hemispherectomies for Sturge-Weber syndrome worldwide. Neurology Dec (1 of 2) 2002;59:1735-1738). (Reprints: Dr Eric H Kossoff, Department of Pediatric Neurology, The Johns Hopkins Hospital, 600 N Wolfe St, Jeffedrson 128, Baltimore, MD 21287).

COMMENT. The main benefit of hemispherectomy in this population is improved seizure control, and the likelihood of seizure freedom is higher when operation is delayed until the child is older.

\title{
CHOROID PLEXUS A-V MALFORMATION PRESENTING WITH IVH
}

A term infant presenting on the second day with apnea and decerebrate posturing had an intraventricular hemorrhage (IVH), the result of an arteriovenous malformation of the choroid plexus demonstrated angiographically on the 3rd day and reported from Johns Hopkins Hospital. The AVM was not demonstrated by ultrasonogram and MR angiogram. Hydrocephalus was managed by acetazolamide and ventricular taps, and a ventriculoperitoneal shunt was placed at 6 weeks. Definitive therapy is delayed until after 1 year. (Heck DV, Gailloud P, Cohen HL et al. Choroid plexus arteriovenous malformation presenting with intraventricular hemorrhage. L Pediatr Nov 2002;141:710-711). (Reprints: Donald V Heck MD, Johns Hopkins Med Ctr, 600 N Wolfe St, Baltimore, MD 21287). 